EPJ Web of Conferences 113,03009 (2016)

DOI: $10.1051 /$ epjconf/201611303009

C) Owned by the authors, published by EDP Sciences, 2016

\title{
Strong interaction studies with kaonic atoms
}

\author{
J. Marton ${ }^{1, a}$, M. Bazzi ${ }^{2}$, G. Beer ${ }^{3}$, C. Berucci ${ }^{1,2}$, D. Bosnar ${ }^{11}$, A.M. Bragadireanu ${ }^{1,5}$, M. Cargnelli ${ }^{1}$,

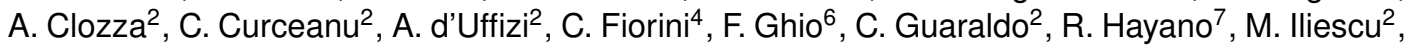 \\ T. Ishiwatari ${ }^{1}$, M. Iwasaki ${ }^{8}$, P. Levi Sandri ${ }^{2}$, S. Okada ${ }^{8}$, D. Pietreanu ${ }^{2,5}$, K. Piscicchia ${ }^{2}$, T. Ponta ${ }^{5}$, \\ R. Quaglia ${ }^{4}$, A. Romero Vidal ${ }^{10}$, E. Sbardella ${ }^{2}$, A. Scordo ${ }^{2}$, H. Shi ${ }^{2}$, D.L. Sirghi ${ }^{2,5}$, F. Sirghi ${ }^{2,5}$, \\ H. Tatsuno ${ }^{12}$, O. Vazquez Doce ${ }^{9}$, E. Widmann $^{1}$, and J. Zmeskal ${ }^{1}$
}

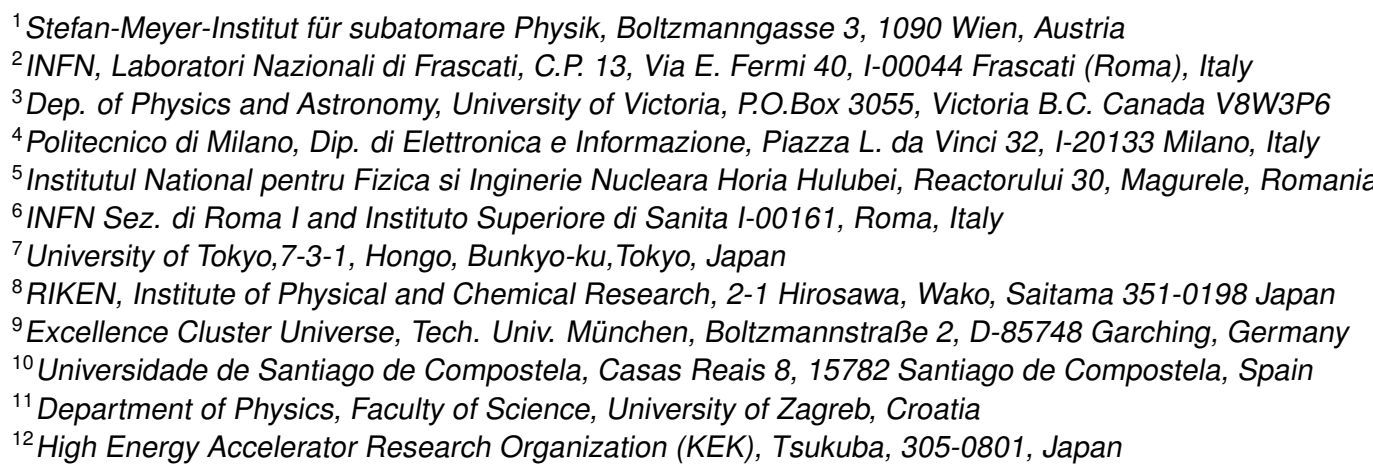

\begin{abstract}
The strong interaction of antikaons $\left(\mathrm{K}^{-}\right)$with nucleons and nuclei in the lowenergy regime represents an active research field connected intrinsically with few-body physics. There are important open questions like the question of antikaon nuclear bound states - the prototype system being $\mathrm{K}^{-} \mathrm{pp}$. A unique and rather direct experimental access to the antikaon-nucleon scattering lengths is provided by precision X-ray spectroscopy of transitions in low-lying states of light kaonic atoms like kaonic hydrogen isotopes. In the SIDDHARTA experiment at the electron-positron collider DAФNE of LNF-INFN we measured the most precise values of the strong interaction observables, i.e. the strong interaction on the $1 \mathrm{~s}$ ground state of the electromagnetically bound $\mathrm{K}^{-} \mathrm{p}$ atom leading to a hadronic shift $\epsilon_{1 s}$ and a hadronic broadening $\Gamma_{1 s}$ of the 1s state. The SIDDHARTA result triggered new theoretical work which achieved major progress in the understanding of the low-energy strong interaction with strangeness. Antikaon-nucleon scattering lengths have been calculated constrained by the SIDDHARTA data on kaonic hydrogen. For the extraction of the isospin-dependent scattering lengths a measurement of the hadronic shift and width of kaonic deuterium is necessary. Therefore, new X-ray studies with the focus on kaonic deuterium are in preparation (SIDDHARTA2). Many improvements in the experimental setup will allow to measure kaonic deuterium which is challenging due to the anticipated low X-ray yield. Especially important are the data on the X-ray yields of kaonic deuterium extracted from a exploratory experiment within SIDDHARTA.
\end{abstract}

a e-mail: johann.marton@oeaw.ac.at 


\section{Introduction}

Hadronic atoms like pionic and kaonic atoms are extremely valuable systems for the investigation of the strong interaction in the low-energy domain.

Especially interesting is the strong interaction involving the strange quark which plays a peculiar role. The strange quark belongs to the light quarks but it is with a mass of about $100 \mathrm{MeV} / \mathrm{c}^{2}$ much heavier than the up and down quarks which have masses in the order of few $\mathrm{MeV} / \mathrm{c}^{2}$. There are data on kaonic atoms available from past experiments (for a review see [1]), however the most simple kaonic atoms like kaonic hydrogen and deuterium are challenging and precision data were obtained in more recent experiments [2-4] using X-ray spectroscopy to unveil the low-energy strong interaction with high precision in these simple systems.

The most basic exotic atom with strangeness represents kaonic hydrogen $\left(\mathrm{K}^{-} \mathrm{p}\right)$ which is an electromagnetically bound exotic atom consisting of a proton and an antikaon $\left(\mathrm{K}^{-}\right)$. In this system one can study the explicit and spontaneous chiral symmetry breaking in a fairly direct way by spectroscopy of $\mathrm{x}$-rays emitted in the transitions to the $1 \mathrm{~s}$ ground state, in this way the threshold data can be deduced. The K-p interaction at threshold is strongly influenced by the sub-threshold resonance $\Lambda(1405)$ which is an interesting hadronic object with a non-trivial nature [5]. In order to deduce the experimental values of isospin-separated antikaon-nucleon scattering lengths one needs the hadronic shift $\epsilon_{1 s}$ and width $\Gamma_{1 s}$ in kaonic hydrogen and kaonic deuterium which can be extracted from the X-ray transitions to the $1 \mathrm{~s}$ ground state in both kaonic systems.

\section{Kaonic hydrogen}

The SIDDHARTA experiment at the DAФNE electron-positron collider succeeded to measure the Xray spectrum of kaonic hydrogen by using an array of silicon drift detectors. From the $\mathrm{K}$ transitions the experimental values of $\epsilon_{1 s}$ and width $\Gamma_{1 s}$ were determined. The energy shift $\epsilon_{1 s}$ is given by the deviation of the measured $\mathrm{K}(\mathrm{np} \rightarrow 1 \mathrm{~s})$ transition energy $\mathrm{E}_{n p \rightarrow 1 s}^{\text {meas. }}$ from the calculated electromagnetic value $\mathrm{E}_{n p \rightarrow 1 s}^{e m}$.

$$
\epsilon_{1 s}=E_{n p \rightarrow 1 s}^{\text {meas. }}-E_{n p \rightarrow 1 s}^{e m}
$$

With a modified Deser formula Equ.2 [6] the antikaon-nucleon scattering length $\mathrm{a}_{p}$ can be calculated which is the averaged sum of the $\mathrm{a}_{0}$ (isospin $\left.\mathrm{I}=0\right)$ and $\mathrm{a}_{1}$ (isospin $\left.\mathrm{I}=1\right)$ scattering lengths $\left(\mathrm{a}_{p}=\right.$ $\left.\frac{1}{2}\left(a_{0}+a_{1}\right)\right)$.

$$
\epsilon_{1 s}+\frac{i}{2} \Gamma_{1 s}=2 \alpha^{3} \mu_{c}^{2} a_{p}\left(1-2 \alpha \mu_{c}(\ln \alpha-1) a_{p}\right)
$$

It is clear that one has to study the antikaon-neutron interaction by using kaonic deuterium to determine the $\mathrm{a}_{0}$ and $\mathrm{a}_{1}$.

\section{Kaonic deuterium}

The case of kaonic deuterium is more challenging than kaonic hydrogen mainly due to the larger widths of the $\mathrm{K}$ lines and the lower X-ray yield expected. In Fig. 1 kaonic deuterium values of $\epsilon_{1 s}$ and $\Gamma_{1 s}$ calculated in different theoretical approaches are displayed.

Experimentally the case of kaonic deuterium is still open. SIDDHARTA measured the X-ray spectrum with a pure deuterium filling but due to the limited statistics and the background condition 


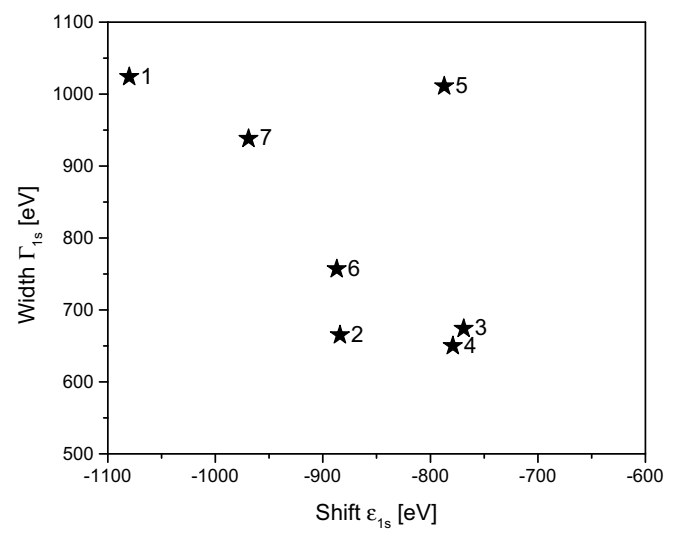

Figure 1. Predicted values of $\epsilon_{1 s}$ and $\Gamma_{1 s}$ obtained in theoretical studies of kaonic deuterium indicated by the numbers 1-7: 1 ref.[7], 2 ref.[6], 3 ref.[8], 4 ref.[9], 5 ref.[10], 6 ref.[11], 7 ref.[12].

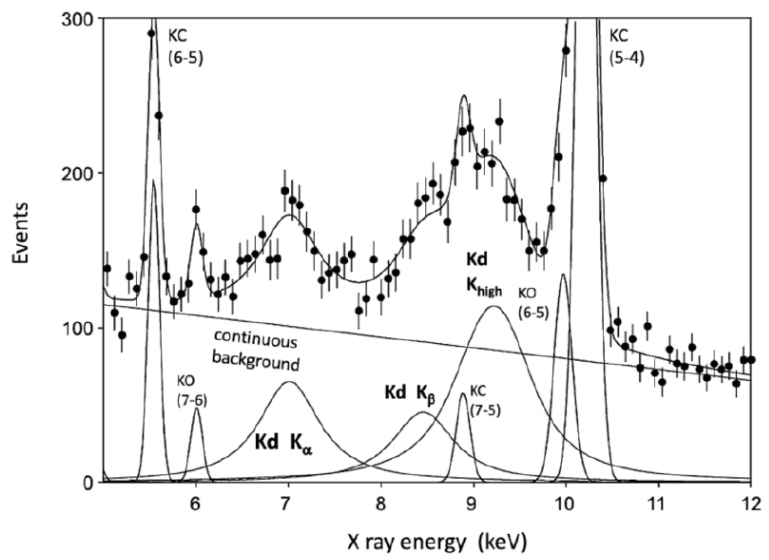

Figure 2. Monte-Carlo calculated X-ray spectrum of kaonic deuterium assuming $\epsilon_{1 s}=-805 \mathrm{eV}$ and $\Gamma_{1 s}=750$ $\mathrm{eV}$ [14]. With this values one gets an estimated precision of $70 \mathrm{eV}$ in the shift and $150 \mathrm{eV}$ in the width.

the determination of $\epsilon_{1 s}$ and $\Gamma_{1 s}$ was impossible. An upper limit for the $\mathrm{X}$-ray yield of the $\mathrm{K}$ lines could be extracted from the data: total yield $<0.0143, \mathrm{~K} \alpha$ yield $<0.0039$ [13].

A new experiment SIDDHARTA2 is planned which is based on a strongly improved apparatus. The improvements include an optimized geometry, deuterium gas density, discrimination of $\mathrm{K}^{+}$, active shielding and better SDD timing performance by cooling. According to Monte Carlo studies one expects an X-ray energy spectrum shown in Fig.2. 


\section{Summary and Outlook}

The SIDDHARTA experiment provided solid data for the antikaon-nucleon interaction at threshold and thus important constraints for the theory of strong interaction with strangeness at low energies. Now a clear description based on an effective field theory with coupled channels is available which is consistent with the information on the antikaon-nucleon interaction [15]. After SIDDHARTA the next important step is the study of the kaonic deuterium X-ray spectrum in order to deduce the isospin separated antikaon-nucleon scattering lengths. This measurement and related studies [16] are crucial for the understanding of strangeness and will provide stringent tests of the theoretical description.

\section{Acknowledgements}

We thank C. Capoccia, G. Corradi, B. Dulach, and D. Tagnani from LNF-INFN; and H. Schneider, L. Stohwasser, and D. Stückler from Stefan-Meyer-Institut, for their fundamental contribution in designing and building the SIDDHARTA setup. We thank as well the DAФNE staff for the excellent working conditions and permanent support. Part of this work was supported by the European Community- Research Infrastructure Integrating Activity "Study of Strongly Interacting Matter" (HadronPhysics2, Grant Agreement No. 227431, and HadronPhysics3 (HP3) Contract No. 283286) under the Seventh Framework Programme of EU; HadronPhysics I3 FP6 European Community program, Contract No. RII3-CT-2004- 506078; Austrian Science Fund (FWF) (P24756-N20); Austrian Federal Ministry of Science and Research BMBWK 650962/0001 VI/2/2009; Croatian Science Foundation under Project No. 1680; Romanian National Authority for Scientific Research, Contract No. 2-CeX 06-11-11/2006; and the Grant-in-Aid for Specially Promoted Research (20002003), MEXT, Japan.

\section{References}

[1] E. Friedman, A. Gal, Phys. Rep. 452, 89 (2007), C.J. Batty, E. Friedman, A. Gal, Phys. Rep. 287, 385 (1997).

[2] M. Iwasaki et al., Phys. Rev. Lett. 78, 3097(1997), T. M. Ito, et al., Phys. Rev. C 58, 2366 (1998).

[3] G. Beer et al., Phys. Rev. Lett. 94, 212302 (2005).

[4] M. Bazzi et al., Phys. Lett. B 704, 113 (2011), Bazzi, M., Beer, G., Bombelli, L., Bragadireanu, A. M., Cargnelli, M. et al. (SIDDHARTA collaboration), Nucl. Phys. A 881, 88 (2012).

[5] T. Hyodo and D. Jido, Prog.Part.Nucl.Phys. 67 (2012) 55-98 arXiv:1104.4474 [nucl-th].

[6] U.-G. Meißner, U. Raha, A. Rusetsky, Eur. Phys. J. C 47, 473 (2006).

[7] S.S. Kamalov, E. Oset, A. Ramos, Nucl. Phys. A 690494 (2001).

[8] A. Gal, Int. J. Mod. Phys. A 22, 226 (2007).

[9] M. Döring, U.-G. Meißner, Phys. Lett. B 704, 663 (2011).

[10] N.V. Shevchenko, Nucl. Phys. A 890-891, 50 (2012).

[11] T. Mizutani, C. Fayard, B. Saghai, K. Tsushima, Phys. Rev. C 87, 035201 (2013).

[12] W. Weise, Hyp. Int. 233, 131 (2015).

[13] M. Bazzi [SIDDHARTA], Nucl. Phys. A 907, 69 (2013).

[14] M. Cargnelli, private communication.

[15] Y. Ikeda, T. Hyodo and W. Weise, Phys. Lett. B 706, 63 (2011).

[16] C. Curceanu et al., Nucl. Phys. A 914, 251 (2013). 